\title{
Cultura digitalizada, buscando estrategias durante la emergencia sanitaria por COVID-19
}

\author{
Digitized culture, seeking strategies during health \\ emergency by COVID-19
}

\author{
María de Lourdes Becerra-Zavala a \\ Julia Josefa Méndez-Delgado ${ }^{b}$ \\ Néstor Igor Hernández-Rivera ${ }^{c}$ \\ Perla Génesis Andrade-Sosa ${ }^{d}$ \\ Zazil Elideth Lozada-Andrade ${ }^{\mathrm{e}}$
}

Recibido: 11 de enero de 2021.

Aceptado: 05 de abril de 2021.

RESUMEN: En México ha pasado un año en aislamiento social en casa debido a la pandemia por COVID-19. A partir de ello surgieron análisis académicos que se suman a las bases de datos oficiales respecto al sector cultural durante la pandemia. En este contexto el Observatorio de Políticas Culturales, adscrito a la Facultad de Antropología de la Universidad Veracruzana, registró datos de

a Observatorio de Políticas Culturales (OPC), Facultad de Antropología, Universidad Veracruzana.

Contacto: Ibecerra@uv.mx | ORCID https://orcid.org/0000-0002-3459-1131

b Observatorio de Políticas Culturales (OPC), Facultad de Antropología, Universidad Veracruzana. Contacto: jujomede.23@gmail.com

c Observatorio de Políticas Culturales (OPC), Facultad de Antropología, Universidad Veracruzana. Contacto: soy.historico.igor@gmail.com

d Observatorio de Políticas Culturales (OPC), Facultad de Antropología, Universidad Veracruzana. Contacto: genesissosa1723@gmail.com

e Observatorio de Políticas Culturales (OPC), Facultad de Antropología, Universidad Veracruzana. Contacto: 
julio a diciembre de 2020 a nivel local y los comparó con actividades convocadas por agentes gubernamentales y académicos. Las artes fueron las de mayor actividad, en contraste con el patrimonio tangible e intangible. Esto corresponde con la tendencia de trabajo de los gestores monitoreados en años anteriores, pero también a que los agentes gubernamentales lanzaron muchas convocatorias enfocadas a esta temática. Es necesario generar información constante que permita saber cómo y por qué regresar a una vida cultural activa, para el diseño e implementación de políticas culturales.

Palabras clave: gestión cultural; COVID-19; políticas culturales; artes; patrimonio.

ABSTRACT: Mexico has spent a year in social isolation at home due to the COVID-19 pandemic. As a result, academic analyses have emerged that add to the official databases regarding the cultural sector during the pandemic. In this context, the Cultural Policies Observatory, attached to the Faculty of Anthropology of the Universidad Veracruzana, recorded data from July to December 2020 at the local level and compared them with activities organized by governmental and academic agents. The arts were the most active, in contrast to tangible and intangible heritage. This corresponds to the work trend of the managers monitored in previous years, but also to the fact that governmental agents launched many calls for proposals focused on this topic. It is necessary to generate constant information that allows us to know how and why to return to an active cultural life, for the design and implementation of cultural policies.

Keywords: cultural managment; COVID-19; cultural policies; arts; heritage.

\section{Introducción}

A un año del inicio del aislamiento social en México, causado por la pandemia mundial por COVID-19, la reorganización de la vida diaria ha generado el surgimiento de nuevas estrategias para las dinámicas escolares, laborales, familiares, recreativas y emotivas. Para el sector cultural, el 2020 ha representado una coyuntura en la que sectores de la sociedad civil han replanteado demandas estructurales, no atendidas suficientemente desde hace años, como la ampliación o reestructuración de programas públicos e incentivos fiscales para creadores y gestores culturales (A un año de "No Vivimos del Aplauso", 2020; Redacción, 2020). También, velozmente, han cobrado mayor presencia demandas relacionadas al papel de la digitalización de procesos culturales (Lara et al., 2020). Esta última ha tenido un papel periférico en los planes nacionales de cultura, ahora más que nunca habría que revalorar su lugar en el diseño de políticas culturales.

Ante este panorama, la Universidad Nacional Autónoma de México (UNAM), a través de la Coordinación de Difusión Cultural, ha publicado estudios a nivel nacional con el propósito de trazar rutas de política cultural. Por un lado, el Estudio sobre Estrategias para el sector cultural hacia el futuro (Cátedra Internacional Inés Amor en Gestión Cultural, 2020), en el que se presentan resultados sobre la situación de salud, financiera, creación y expectativas de los trabajadores culturales. Otro fue la Encuesta Nacional sobre Hábitos y Consumo Cultural 2020 (Difusión Cultural UNAM, 2021), a partir de la cual se analizan intereses, hábitos durante la pandemia, estado de 
ánimo, perspectivas hacia los siguientes meses, temas de atención prioritaria para la vuelta a espacios y actividades culturales, dificultades enfrentadas por los gestores en aislamiento.

Los análisis arriba mencionados son valiosos porque complementan la información registrada por el Instituto Nacional de Estadística y Geografía (INEGI) desde la Cuenta Satélite de Cultura, y la Encuesta Nacional de Hogares. Así, es necesario ampliar la información sobre los mismos aspectos en contextos regionales y locales. El Observatorio de Políticas Culturales, adscrito a la Facultad de Antropología de la Universidad Veracruzana (OPC-FAUV), realizó el monitoreo regular hecho desde el 2018 con los gestores de Recultivar México, además de uno especial sobre actividades propuestas por instituciones gubernamentales de cultura y grupos de trabajo con al menos 3 años de actividades ininterrumpidas, y que tuvieran un sitio web.

\section{Metodología}

La política cultural es una política pública en la que intervienen agentes culturales gubernamentales de la iniciativa privada y la sociedad civil. Estos interactúan a partir de la definición de sus objetivos, normas legales y socioculturales, en procesos de mediano alcance en un territorio definido (Nivón Bolán, 2012). Con la intención de identificar algunas interacciones entre agentes, el OPC-FAUV registró las actividades convocadas en Facebook por gestores culturales miembros de Recultivar México, ubicados predominantemente en la región centro del estado de Veracruz. De julio a diciembre 2020, 29 de los 60 tuvieron actividad. En un monitoreo especial, se registraron actividades convocadas desde los sitios web de la Secretaría de Cultura, Instituto Veracruzano de Educación y Cultura, la Universidad Veracruzana, Grupo de Reflexión sobre Economía y Cultura, Observatorio Cultural Ciudadano de Veracruz y la Red Universitaria de Gestión Cultural México.

Las actividades se han categorizado por dominios, los cuales representan la cultura por áreas caracterizadas en un grupo común recomendado por la Oficina de las Naciones Unidas para la Educación, la Ciencia y la Cultura (Instituto de Estadística de la UNESCO, 2014) para recuperar y comparar datos culturales. Las áreas se relacionan con procesos vinculados a los indicadores de gestión cultural y derechos culturales, como el tipo de participación a la que convocan (talleres, exposiciones, festivales, etc.) y el objetivo del evento (divulgar, educar, etc).

A continuación, se presenta el análisis de la información para reflexionar sobre los temas culturales que han predominado en este año, las condiciones en las que los gestores culturales se mantuvieron activos a lo largo de 2020, así como las herramientas digitales han ocupado.

\section{Resultados y discusión}

\subsection{Plataformas virtuales}

En total, se realizaron 650 actividades culturales en el monitoreo especial de cultura y COVID-19 desde agosto hasta diciembre de 2020. En la tabla 1 se pueden observar las plataformas digitales han sido utilizadas: 


\section{Tabla 1. Plataformas utilizadas para realizar actividades virtuales en el monitoreo} especial. Agosto-diciembre 2020

Espacio virtual utilizado IDR

Número de actividades

\begin{tabular}{lc}
\hline Facebook, Facebook live & 337 \\
\hline Blog o sitio web & 129 \\
\hline $\begin{array}{l}\text { Utilizan al menos dos de las codificadas para transmitir } \\
\text { simultáneamente }\end{array}$ & 106 \\
\hline Youtube & 27 \\
\hline Zoom & 26 \\
\hline Canal de televisión & 14 \\
\hline Instagram & 1 \\
\hline Google meet & 1 \\
\hline Sin datos & 9 \\
\hline Total general & $\mathbf{6 5 0}$ \\
\hline
\end{tabular}

Fuente: OPC-FAUV, 2020.

Hay congruencia con la Encuesta de hábitos y consumo cultural (Difusión Cultural UNAM, 2021) en cuanto a los espacios virtuales utilizados: después de WhastApp, Facebook es la red social más utilizada por las personas. Eso puede indicar que mucha actividad cultural se trasladó a este espacio, pues ya se habían conformado comunidades virtuales desde antes de la pandemia, las cuales se vieron aumentadas o consolidadas durante el periodo de aislamiento social.

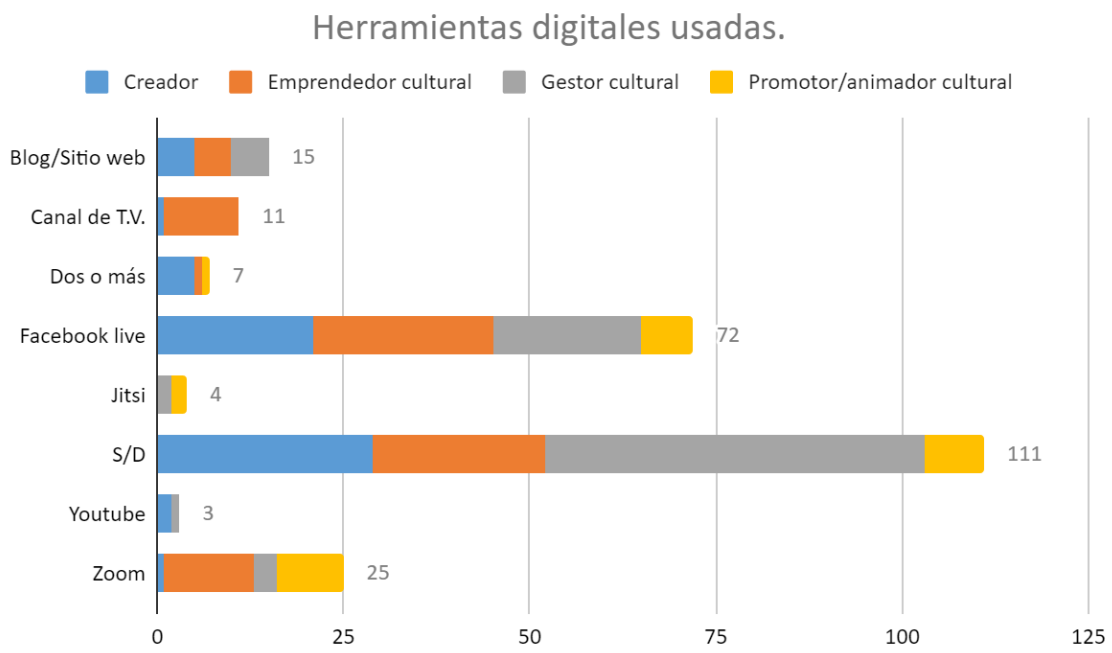

Figura 1. Plataformas utilizadas por Gestores Recultivar México. julio-diciembre 2020. Fuente: OPC-FAUV, 2020.

Por otro lado, los gestores de Recultivar México también han hecho de Facebook un nuevo espacio de comunicación social para abrirse paso en los medios digitales. Cabe señalar que las actividades que se registraron Sin Datos (S/D) indican que no se señalaron espacios virtuales de realización y que en realidad se llevaron a cabo de manera presencial con protocolos sanitarios. Ello corresponde 
a algunos espacios culturales independientes (restaurantes que son foros culturales) y espacios relacionados al patrimonio natural.

\subsection{Dominios y agentes culturales}

En el monitoreo especial, las artes fueron las de mayor presencia, particularmente la música, pues ocupó 138 eventos (casi dos terceras partes del total de actividades artísticas). Altamente valoradas por los agentes gubernamentales, la Universidad Veracruzana y grupos de investigación ocupan más de la mitad del total de actividades registradas. En segundo lugar, se presentaron actividades de carácter educativo (cursos, talleres, divulgación). Los patrimonios ocuparon un tercero y cuarto lugar, sin embargo, si sumamos las actividades registradas de patrimonios y educación, estas no llegan ni a la mitad de actividades artísticas registradas.

\section{Tabla 2. Ámbitos culturales con actividades virtuales registradas en el Monitoreo Especial. Agosto-diciembre 2020}

\begin{tabular}{|c|c|}
\hline Dominio cultural & Número de actividades \\
\hline Artes & 430 \\
\hline Educación & 100 \\
\hline Patrimonio Intangible & 58 \\
\hline Patrimonio Tangible & 57 \\
\hline Patrimonio Natural & 5 \\
\hline Total general & 650 \\
\hline
\end{tabular}

Fuente: OPC-FAUV, 2020.

En cuanto los gestores Recultivar México, las actividades registradas de julio a diciembre 2020 atendieron los siguientes temas:

Tabla 3. Actividades de gestores Recultivar México. Julio-diciembre 2020

\begin{tabular}{|c|c|}
\hline Dominio cultural & Número de actividades \\
\hline Artes & 122 \\
\hline Educación & 56 \\
\hline Patrimonio Natural & 56 \\
\hline Patrimonio Intangible & 20 \\
\hline Patrimonio Tangible & 0 \\
\hline Total general & 254 \\
\hline
\end{tabular}

Fuente: OPC-FAUV, 2020.

Hubo pocas actividades sobre patrimonio intangible difundidas en las plataformas virtuales frente al resto de las temáticas. Se realizaron varias acciones entorno al patrimonio natural, favoreciendo la participación colectiva activa. Alimentación saludable, huertos comunitarios, restauración de áreas naturales, fueron algunas de las formas de convocar tanto virtual como presencialmente. 
El patrimonio tangible ha quedado rezagado en la lista de intereses de los gestores Recultivar. Esto no es nuevo, en años anteriores había sido semejante pero no se habían registrado cero actividades. Esto puede corresponder a que la gestión y salvaguarda es legalmente tarea casi exclusiva del gobierno federal en nuestro país, pero también es menester considerar que, de acuerdo a la Encuesta de hábitos y consumo cultural (Difusión Cultural UNAM, 2021), ir a sitios arqueológicos estaba entre las últimas 3 de 12 actividades preferidas para el tiempo libre antes de la pandemia. Es decir: no había un hábito consolidado que se viera favorecido en condiciones de aislamiento.

También está el hecho de vivir una experiencia física, visual, conviene señalar que este tipo de experiencia se da en el patrimonio tangible ya sea con esculturas o con estructuras de carácter arqueológico. Se coincide con lo planteado por Nivón Bolán (2021) en que la digitalización de recorridos virtuales o del patrimonio tiene sus límites y requiere de estrategias educativas cuidadosamente planteadas para despertar el interés en las audiencias.

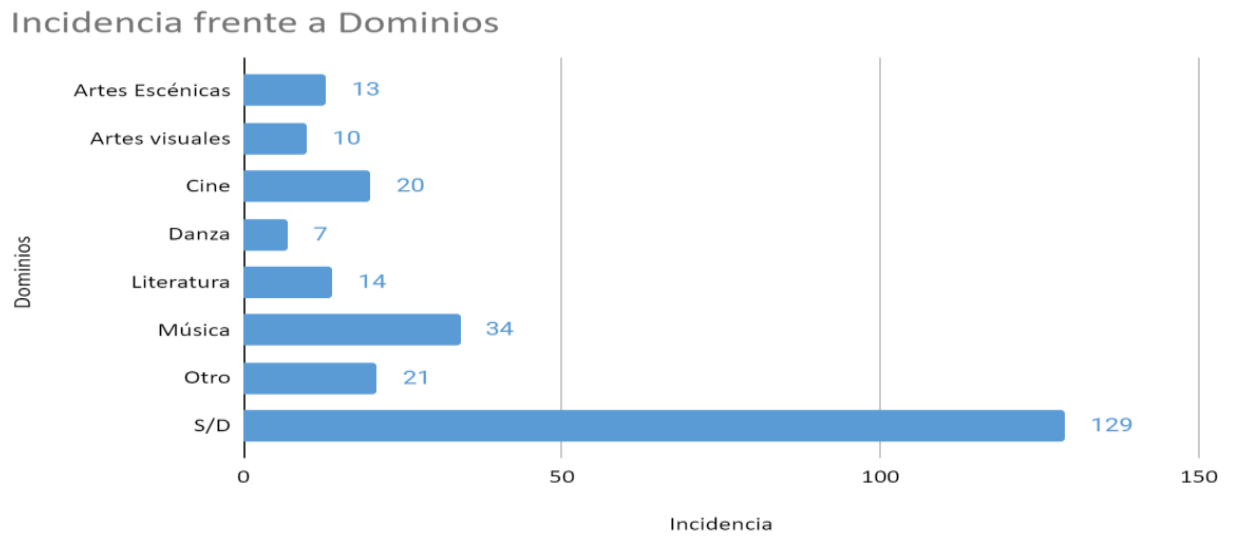

Figura 2. Incidencia de actividades artísticas de gestores Recultivar México frente a otros dominios culturales. Julio-diciembre 2020. Fuente: OPC-FAUV, 2020.

De nuevo, las artes ocupan el principal interés de los gestores Recultivar México, especialmente la música, el cine y las artes escénicas. Esta era una tendencia antes de la pandemia (Observatorio de Políticas Culturales, 2019). El público se inclina hacia estas actividades en el tiempo libre, pero esto ya era una preferencia antes de la pandemia (Difusión Cultural UNAM, 2021). Ir al cine, a conciertos son 2 de 3 actividades que se realizaban antes de 2020. No obstante, ir a museos disminuyó durante la cuarentena, a pesar de que estaba entre las 3 predilectas.

La Encuesta de hábitos y consumo cultural (Difusión Cultural UNAM, 2021) registró que, en la cuarentena, escuchar música o ver videos musicales lo realiza diariamente el $70.3 \%$ de los encuestados. Si bien en este periodo se han diversificado los intereses de las audiencias hacia el teatro o la danza (Nivón Bolán, 2021) es necesario reflexionar en qué medida los agentes que abren convocatorias y ofrecen actividades en línea pueden consolidar nuevas audiencias en relación con los procesos de creación y producción para las artes. 


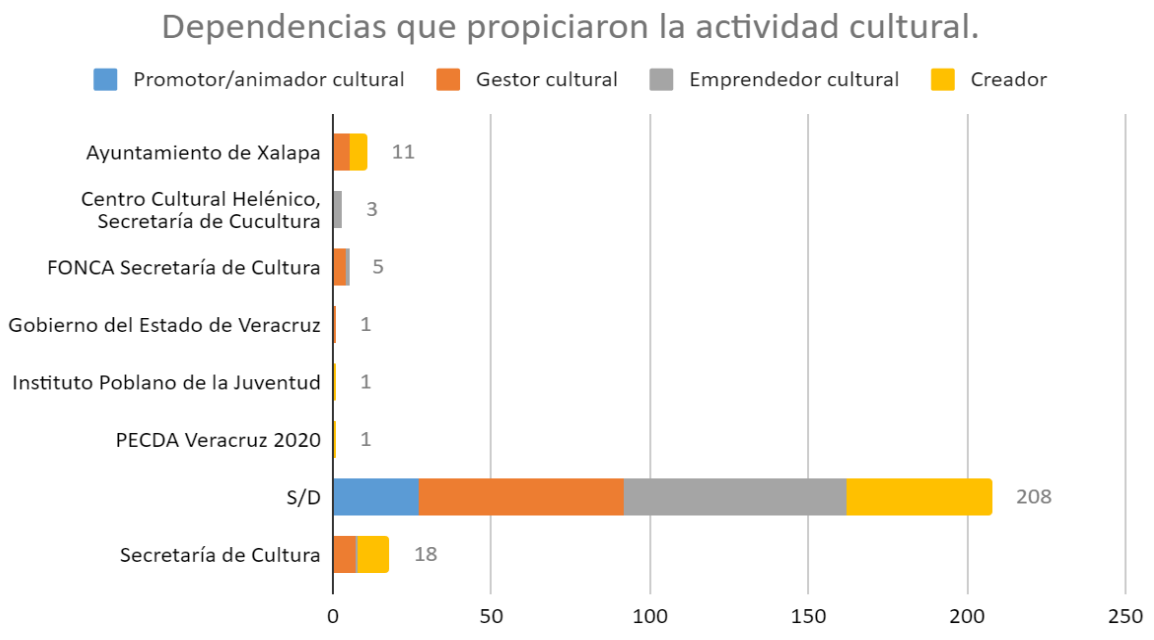

Figura 3. Colaboradores gubernamentales de los gestores Recultivar México. Agosto-diciembre 2020. Fuente: OPC-FAUV, 2020.

Las dependencias gubernamentales federales o municipal (en Xalapa) ofrecieron mayores impulsos (con o sin convocatoria de por medio). Los meses de octubre y noviembre fueron los de mayor actividad para los gestores Recultivar. El aumento se llevó a cabo junto con las nuevas convocatorias lanzadas por gobiernos estatal y federal a lo largo del año, las cuáles se han visto modificadas después de la pandemia por COVID-19.

\section{Conclusiones}

Durante este confinamiento las instituciones de gobierno, educativas y agentes de la sociedad civil invitan a experimentar nuevas formas para seguir juntos. Estas prácticas han apostado por el uso de las herramientas digitales como estrategias para mantener la comunicación a través de medios virtuales.

Se destaca que las actividades artísticas tienen una gran presencia dentro de las políticas culturales. Es prudente preguntarse: ¿por qué han tenido un mayor impacto dentro del espacio virtual? Algunas de ellas ya estaban consolidadas como habituales o eran preferidas antes de la pandemia, sin embargo, las políticas culturales, sean de carácter público gubernamental o desde la sociedad civil, deberían fomentar la presencia de otros ámbitos de la vida creativa y cultural. A partir de una serie de peticiones por parte del sector cultural, el gobierno incrementó y redireccionó muchas convocatorias para las artes. En contraste, el patrimonio cultural de México se ha visto afectado. Esto es particularmente relevante para Veracruz, considerando la presencia de tres Patrimonios de la Humanidad, una Reserva de Biósfera, y la diversidad cultural de las poblaciones originarias y afromestizas en 212 municipios.

Aun cuando aumentó la digitalización de acervos museísticos, de galerías y de otros espacios culturales del país esto no necesariamente se refleja en el interés por parte de las audiencias. Las herramientas digitales no hacen por sí mismas espacios sociales virtuales y su uso debe 
reconsiderarse en términos de educación y creación de públicos si es que se desea incorporarlos seriamente a las políticas culturales, ya sea para una implementación virtual o presencial.

En 2021 se requiere saber cómo regresar a una vida cultural activa, tanto para organizaciones sin fines de lucro, dependencias públicas, emprendedores culturales que se han visto afectados seriamente, como para quienes participan de esa experiencia. El papel de los agentes estatales y municipales será fundamental porque las dependencias federales no pueden ni deberían atender solas la diversidad de intereses culturales en cada municipio. Por ello la generación constante de información pertinente para evaluación, diseño e implementación de políticas culturales, es una tarea constante para académicos, gestores y gobierno.

\section{Referencias}

A un año de "No Vivimos del Aplauso". (2020, diciembre 24). A un año de "No Vivimos del Aplauso", la lucha en contra de la precarización laboral ante la pandemia. Infobae. /america/entretenimiento/2020/12/24/a-un-ano-de-no-vivimos-del-aplauso-lalucha-en-contra-de-la-precarizacion-laboral-ante-la-pandemia

Cátedra Internacional Inés Amor en Gestión Cultural. (2020). Para salir de terapia intensiva. Estrategias para el sector cultural hacia el futuro. Cultura UNAM. https://bit.ly/3axMH5b

Difusión Cultural UNAM. (2021). Encuesta Nacional de hábitos y consumo cultural. Análisis cualitativo y estadístico. UNAM. https: //bit.ly/3gtExhX

Instituto de Estadística de la UNESCO. (2014). Cómo medir la participación cultural. Manual del Marco de Estadísticas Culturales de la UNESCO. 2009. №2. UNESCO. https://bit.ly/32Ej65x

Lara, C., Bravo, J., Negrete, J. F., \& Hermosillo, J. M. (2020, julio 18). Política Digital para la Cultura: Una primera dosis para el Covid-19. El Universal. https://bit.ly/3ay0x7g

Observatorio de Políticas Culturales. (2019). Boletines Enero/Julio y Agosto/Diciembre 2019 [Académica]. Observatorio de Políticas Culturales de la Facultad de Antropología. https://bit.ly/3gEDVWy

Nivón-Bolán E. (2012). Desarrollo y debates actuales de las políticas culturales en México. Universidad Autónoma Metropolitana, Iztapalapa. https://bit.ly/32FjmkY

Nivón-Bolán, E. (2021, febrero 8). Cultura UNAM: Cambia Covid Hábitos y consumo cultural [Paso Libre. Grecu]. Cultura y Política. https://bit.ly/3sIUH9A

oRedacción. (2020, marzo 23). Red de espacios culturales independientes pide apoyo contra la crisis por Covid-19. La Jornada. https://bit.ly/32C3Iqt 